\title{
A general theory of structure spaces
}

by

\section{A. Holme (Oslo)}

Structure spaces have been considered for a great number of different algebraic systems such as rings, Banach algebras, semi-rings, lattices, lattice-ordered groups etc. In a situation like this the problem of unification naturally poses itself. The purpose of this paper is to show that the theory of $x$-ideals as developed in [1] and [2] appears as a natural framework for a general theory of structure-spaces. Some indications in this direction were already given in [1]. We shall here pursue the subject along more general lines. The present development is in fact general enough to cover a large number of special cases. On the other hand a reasonable part of the theory of structure spaces for special algebraic systems may be generalized to our situation.

In $\S 0$ we first give the necessary algebraic background. With exception of Proposition 0.2 this is contained in [1] and [2], to which we refer for proofs, more details and special cases. In $\S 1$ we turn to the theory of structure-spaces for commutative semi-groups with an $x$-system. Theorems 1,2 and 3 concerning Hausdorff structure-spaces are mainly generalizations of parts of the corresponding theory for rings in [4]. In $\S 2$ we consider the compactness of structure-spaces. The theory there follows the same lines as the theory in [3] and [5]. It is easy to see that if $R$ is a semisimple commutative ring with identity, then $\mathfrak{M}(R)$ is disconnected if and only if $R$ is the direct sum of two of its ideals, $R_{1}$ and $R_{2}$. If $\mathfrak{M}(R)=\mathfrak{F}_{1} \cup \mathfrak{F}_{2}$ is a partition of $\mathfrak{M}(R)$ into disjoint open-closed proper subsets, $R_{1}$ and $R_{2}$ may be chosen so that $\mathfrak{M}\left(R_{i}\right)$ is homeomorphic to $\mathfrak{\mho}_{i}, i=1,2$. The argument here makes use of the additive structure of the ring. In $\S 3$ we show how this may nevertheless be transferred to our situation, the additivity being taken care of mainly by the additivity axiom for an $x$-system on a commutative semigroup, introduced in [2]. The representation theorem of $\S 4$ is related to Theorem 14 of [10]. Finally, we illustrate by a simple example a rather interesting procedure: an algebraic problem may be reformulated in terms of structure-spaces, and solved by simple topological reasoning.

I should like to thank Professor K. E. Aubert for his help during the preparation of the present paper. 
\$ 0. Preliminaries. Let $S$ be a commutative semigroup. We shall say that there is defined an $x$-system on $S$ if to every $A \subseteq S$ there corresponds $A_{x} \subseteq S$ such that

$$
\begin{gathered}
A \subseteq A_{x}, \\
A \subseteq B_{x} \Rightarrow A_{x} \subseteq B_{x}, \\
A B_{x} \subseteq B_{x}, \\
A B_{x} \subseteq(A B)_{x} .
\end{gathered}
$$

$(0.4)$ is referred to as the continuity axiom. A subset $A$ in $S$ is said to be an $x$-ideal, or shorter an ideal if $A=A_{x}$.

We may give an equivalent definition of an $x$-system in the following way: let $S$ be a commutative semigroup, and let $\mathfrak{X}$ be a non-empty family of subsets of $S$, called $x$-ideals, such that the following conditions are satisfied:

(0.5) The intersection of any non-empty family of $x$-ideals is an $x$-ideal.

(0.6) For any $a \in S$ and any $A \in \mathfrak{X}, A: a$ is an $x$-ideal containing $A$.

Let $A$ be any subset of $S$, and put $A_{x}=\bigcap_{\substack{B \in\{ \\A \subset B}} B$. Then the correspondence $A \rightarrow A_{x}$ defines an $x$-system in the sense of (0.1)-(0.4) and the family of $x$-ideals is $\mathfrak{X}$.

$A_{x}$ is said to be a proper $x$-ideal if $A_{x} \neq S, \varnothing$. A prime $x$-ideal is an ideal $P_{x}$ satisfying $a b \in P_{x} \Rightarrow a \in P_{x} \vee b \in P_{x}$, and a maximal $x$-ideal is a proper ideal not properly contained in any proper ideal. A minimal prime ideal is defined correspondingly. The families of prime, maximal and minimal ideals in $\mathbb{S}$ are denoted by $\mathfrak{P}, \mathfrak{M}$ and $\mathfrak{N}$ respectively.

An $x$-system is said to be of finite character if the set-theoretic union of any chain of $x$-ideals is an $x$-ideal.

If $S$ is a commutative semigroup with an $x$-system, and $T$ is a subsemigroup of $S$, then it is easily verified that the family of all intersections between an $x$-ideal in $S$ and $T$ defines an $x$-system on $T$. This $x$-system is said to be induced on $T$ from $S$. (This definition is not the best one, but will be the relevant definition for our purpose.)

Given a family of $x$-ideals in $S,\left\{A_{x}^{(i)}\right\}_{i \in I}$. Put $\bigcup_{i \in I} A_{x}^{(i)}=\left(\bigcup_{i \in I} A_{x}^{(i)}\right)_{x}$. $\mathfrak{X}$ is a complete lattice under $\cup_{x}$ and $n$. $\cup_{x}$ will be referred to as $x$-union. Furthermore, put $A_{x}{ }^{\circ} B_{x}=(A B)_{x}$. This operation is referred to as $x$-multiplication.

The nilpotent radical of an $x$-ideal $A_{x}$, denoted by $\operatorname{rad} A_{x}$, is the set of all elements $a$ in $S$ such that for some $n, a^{n} \in A_{x} . A_{x}$ is said to be half-prime if $\operatorname{rad} A_{x}=A_{x}$. If every $x$-ideal in $S$ is half-prime, the $x$-sys- tem is said to be half-prime. The element $e \in S$ is called an $x$-identity if $(e)_{x}=S$ and $e \in S^{2}$.

An $x$-ideal $A_{x}$ is shown to be non-prime if and only if $A_{x} \supseteq B_{x}{ }^{\circ}{ }_{x} C_{x}$ for some $B_{x}$ and $C_{x}$ properly containing $A_{x}$. This implies that if $S$ has an $x$-identity, then $\mathfrak{M} \subseteq \mathfrak{P}$.

A subset $M$ of $S$ which is either empty or closed under multiplication is referred to as an $m$-set. We have, for $x$-systems of finite character:

Proposition 0.1. Given an $x$-ideal $A_{x}$ in S. If $M$ is a maximal m-set contained in $C A_{x}$, and $P_{x}$ is an ideal maximal with respect to the property of containing $A_{x}$ and being contained in $\mathrm{C} M$, then $P_{x}$ is a minimal prime ideal over $A_{x}$. (C $A_{x}$ denotes the complement of $A_{x}$ in $S$.)

Corollary 1. Any prime ideal $P_{x}$ over $A_{x}$ contains a least one minimal prime ideal over $A_{x}$.

Corollarr 2. For any maximal m-set $M$ contained in $\mathrm{C} A_{x}, \mathrm{C} M$ is a minimal prime ideal over $\mathcal{A}_{x}$.

For a number of algebraic systems, the following condition turms out to be of importance:

(0.7) To every $a \in S$ there exists an idempotent element $|a|$ such that for every $x$-ideal $A_{x}$ in $S, a \in A_{x} \Leftrightarrow|a| \in A_{x}$.

For a distributive lattice with the $l$-system, we may choose $a=|a|$, for a lattice ordered group with the $c$-system and the multiplication $a \circ b=|a| \cap|b|$, we put $|a|=a \vee 0-a \wedge 0$.

At present, we shall only note the following consequence of $(0.7)$, which will be usefull later (see [7], Theorem 6.5):

Proposition 0.2. Given an ideal $A_{x} \neq \emptyset$, in an $x$-system satisfying (0.7). Then an ideal $P_{x} \supseteq A_{x}$ is a minimal prime ideal over $A_{x}$ if and only if it satisfies the following condition:

(0.8) To every idempotent element $c$ in $P_{x}$ there exists $b \notin P_{x}$ such that $c b \in A_{x}$.

Proof. If $P_{x}$ satisfies (0.8) and $A_{x} \subseteq Q_{x} \subsetneq P_{x}$ for a prime ideal $Q_{x}$, choose $a \in P_{x}, a \notin Q_{x}$. Then $|a| \in P_{x},|a| \notin Q_{x}$. To $|a|$ there corresponds by (0.8) $b \notin P_{x}$ such that $|a| \cdot b \in A_{x}$. Thus $|a| \epsilon Q_{x}$, a contradiction. Conversely, if $P_{x}$ is a minimal prime ideal over $A_{x}, M=\mathrm{C} P_{x}$ is an $m$-set, maximal in $\mathrm{C} A_{x}$ by Corollary 2 above. For an idempotent element $c$ in $A_{x}$, put $M(c)=M \cup\{b c ; b \in M I$. Obviously $M(c)$ is an $m$-set properly containing $M$, thus $M\left(C^{\prime}\right) \cap A_{x} \neq \emptyset$ and $(0.8)$ is satisfied.

THEOREM 0.1 (Krull-Stone). Fror an $x$-system of finite character, $\operatorname{rad} A_{x}$ is equal to the intersection of the minimal prime ideals over $A_{x}$.

Let $S$ and $T$ be commutative semigroups with $x$-systems which we denote respectively by $y$ and $z$. We shall say that a multiplicative F undamenta Mathematicae, T. LVIII 
homomorphism $\varphi$ of $S$ into $T$ is a $(y, z)$-homonorphism if $\varphi\left(A_{y}\right) \subseteq(\varphi(A))_{x}$, for all subsets $A$ of $S$, or, equivalently, if the inverse image of a $z$-ideal in $T$ is a $y$-ideal in $S$. If $\varphi$ is a multiplicative homomorphism of a commutative semigroup $S$ onto a semigroup $T$, and $S$ has an $x$-system $y$, then the set of all $B \subset T$ such that $\varphi^{-1}(B)$ is a $y$-ideal in $S$ defines an $x$-system $y_{\varphi}$ in $T$. This makes $p$ to a $\left(y, y_{\varphi}\right)$-homomorphism.

For $a, b \in S$, put

$$
a \equiv b\left(A_{x}\right) \Leftrightarrow\left(A_{x}, a\right)_{x}=\left(A_{x}, b\right)_{x} .
$$

This is a congruence relation in $S$, which we refer to as $x$-congruence. If $\varphi$ denotes the canonical multiplicative homomorphism of $S$ onto $S / A_{x}$, we shall call $\varphi_{x}$ the canonical $x$-system on $S / A_{x}$.

The $x$-system on $S$ is said to be additive if for any $x$-ideals $A_{x}, B_{x}$ in $S$ and $c \in A_{x} \cup_{x} B_{x}$, there exists $b \in B_{x}$ such that $c \equiv b\left(A_{x}\right)$.

THEOREM 0.2. An $x$-system on $S$ is additive if and only if the canonical mapping $\left.\left.A_{x}\right|_{A_{x} \cap B_{x}} \rightarrow A_{x} \cup_{x} B_{x}\right|_{B_{x}}$ is bijective for any $x$-ideals $A_{x}$ and $B_{x}$ in $S$.

Given any family $\mathfrak{I}$ of $x$-ideals in $S$, we adopt the following notation: For $\mathfrak{A} \subseteq \mathfrak{I}$, put $k \mathfrak{U}=\bigcap_{x} A_{x}$, for an $x$-ideal $B_{x}$ in $S, h B_{x}$ $=\left\{A_{x} \in \mathfrak{I} ; B_{x} \subseteq A_{x}\right\}$. Furthermore, put $k \mathfrak{X}=O_{x}$.

\$1. Structure spaces and their separation properties Let $S$ be a commutative semigroup with an $x$-system, and let $\Im \subseteq \mathfrak{X}$ be a family of proper $x$-ideals such that

$$
B_{x} \cap C_{x} \subseteq A_{x} \Rightarrow B_{x} \subseteq A_{x} \vee O_{x} \subseteq A_{x}
$$

whenever $A_{x} \in \Im$ and $B_{x}$ and $C_{x}$ are arbitrary intersections of ideals from $\mathfrak{I}$. Under this assumption $\mathfrak{I}$ is said to be a structure-family for $\mathbb{S}$.

For $\mathfrak{A} \subset \mathfrak{J}$, put $\overline{\mathfrak{A}}=h \mathfrak{A} \mathfrak{A}$ if $\mathfrak{A} \neq \emptyset, \bar{\emptyset}=\emptyset$.

The above definitions are justified by

Proposition 1. $\mathfrak{U} \rightarrow \overline{\mathfrak{A}}$ defines a topology on $\mathfrak{I}$ if and only if $\mathfrak{I}$ is a structure-family for $S$.

Proof. (1.1) is equivalent to $\overline{\mathfrak{A} \cup \mathfrak{B}} \subseteq \overline{\mathfrak{A}} \cup \overline{\mathfrak{B}}$ for $\mathfrak{A}, \mathfrak{B} \subseteq \mathfrak{I}$. As $\mathfrak{A} \subseteq \overline{\mathfrak{A}}$, $\overline{\mathfrak{A}}=\overline{\mathfrak{A}}$ and $\overline{\mathfrak{A}} \cup \overline{\mathfrak{B}} \subset \overline{\mathfrak{A} \cup \mathfrak{B}}$ are satisfied, without assuming (1.1), the proposition follows.

This topology is referred to as the Zaryski topology and also as the Stone topology on $\mathfrak{I}$, the corresponding topological space is called a structure-space for $S$. It will be denoted by $\mathfrak{I}(\mathbb{S})$.

There is an obvious 1-1 correspondence between the subfamilies of a structure-family and the subspaces of the corresponding structurespace, in the sense that every sub-family of a structure-family is a structure-family, and all subspaces are obtained in this way.
$\mathfrak{P}$ is a structure-family for $S$. If $S$ has an $x$-identity, $\mathfrak{M} \subseteq \mathfrak{P}$, and consequently $\mathfrak{M}$ is a structure-family for $S$.

A structure-space is invariant under $(y, z)$-isomorphisms, and every closed subset of a structure-space for $S$ is homeomorphic to a structurespace for a $(y, z)$-homomorphic image of $S$ :

Proposimion 2. Let $S, T$ be commutative semigroups, with $x$-systems, denoted by $y, \approx$ respectively. Let $\mathfrak{I}$ be a structure-family for $S, \varphi \quad a(y, z)$ isomorphism of $S$ onto $T$, and put $\varphi(\mathfrak{I})=\left\{\varphi\left(A_{y}\right) ; A_{y} \in \mathfrak{I}\right\}$. Then $\varphi(\mathfrak{I})$ is a structure-family for $T$, and $\Im(S)$ is homeomorphic to $(\varphi(\Im))(T)$.

For $A_{y} \subseteq S$, put $\widetilde{\mathfrak{J}}=\left\{B_{y} ; B_{y} \in \mathfrak{I}\right.$ and $\left.B_{y} \supseteq A_{y}\right\}$.

Then $\widetilde{\Im}$ is a closed subset of $\Im(S)$, and is homeomorphic to $(\psi(\Im))\left(S / A_{y}\right)$ where $\psi$ is the canonical homomorphism of $S$ onto $S / A_{y}$. Conversely, if $\mathfrak{F} \subseteq \mathfrak{J}(S)$ is closed, $\mathfrak{F}=\widetilde{J}$ where $A_{y}=k \mathfrak{F}$.

Proof. The first part of the proposition follows from the fact that structure-families and structure-spaces are defined by properties invariant under $(y, z)$-isomorphisms. Clearly, $\widetilde{\mathfrak{F}}$ is closed in $\mathfrak{I}(S)$. Put $\Phi\left(B_{y}\right)$ $=\psi\left(B_{y}\right)$. We hare

$$
\begin{aligned}
B_{y} \supseteq C_{y} & \Leftrightarrow \psi\left(B_{y}\right) \supseteq \psi\left(C_{y}\right) \quad \text { for every } \quad B_{y} \in \widetilde{\Im}, C_{y} \in \widetilde{\Im}, \\
\psi\left(\bigcap_{k \in K} C_{y}^{(k)}\right) & =\bigcap_{k \in K} \psi\left(C_{y}^{(k)}\right), \quad \text { where } \quad C_{y}^{(k)} \epsilon \widetilde{I} \text { for every } k \in K .
\end{aligned}
$$

It follows at once that $\psi(\widetilde{\mathfrak{S}})$ is a structure-family for $S / A_{y}$ and that $\Phi: \widetilde{\Im}(S) \rightarrow \psi(\widetilde{\mathfrak{J}})\left(S / A_{y}\right)$ is a homeomorphism. The last part of the proposition is obvious.

CoRollary. Assume that $\mathfrak{M}$ is a structure-family for $S$, and let $\mathfrak{F}$ be a closed subset of $\mathfrak{M}\left(S^{\prime}\right)$. Then the family $\mathfrak{M}^{\prime}$, of maximal ideats in $S / k \mathfrak{F}$, is a structure-family for $\mathbb{S} / k \mathfrak{F}$, and $\mathfrak{M}^{\prime}(\mathbb{S} / k \mathfrak{F})$ is homeomorphic to $\mathfrak{F}$.

Proof. This follows from Proposition 2 with $\mathfrak{M}^{\prime}=\psi(\mathfrak{F})$ and $\psi: S \rightarrow S^{\prime} / k \widetilde{Y}$ (the canonical homomorphism).

(1.4) For $a \in \mathbb{S}$, put $\mathfrak{F}(a)=h\{a\}, \quad \mathfrak{U}(a)=\mathrm{C} \mathfrak{F}(a)$

Proposimion 3. $\{\mathfrak{U}(a)\}_{a \in S}$ constitutes a basis for the topology on $\mathfrak{I}(S)$. Proof. Since $\mathfrak{F}(a)$ is closed, $\mathfrak{U}(a)$ is open. For $\mathfrak{U}$ open in $\mathfrak{I}(S)$ and $A_{x} \in \mathfrak{U}$, we may find $a \in S$ such that $a \notin A_{x}, a \in \mathbb{C}$ CU. Then $A_{x} \in \mathfrak{U}(a) \subset \mathfrak{U}$.

For the study of separation properties of a structure-space $\mathfrak{I}(S)$, we introduce, for $A_{x} \in \mathfrak{I}(S)$,

$$
N\left(A_{x}\right)=\bigcup_{\left\{b \mid b \in A_{x}\right\}}\left[\bigcap_{\left\{B_{x} \in \mathfrak{S} \mid b \notin B_{x}\right\}} B_{x}\right] .
$$

By Lemma 1 below this definition coincides with Definition 2.1 of [4] if $S$ is a ring with the usual ideal system. 
LEMMA 1. Let $\mathfrak{B}$ be a basis for the neighbourhood system of $A_{x}$. Then

$$
N\left(A_{x}\right)=\bigcup_{\mathfrak{U} \in \mathfrak{B}} k \mathfrak{U} \mathfrak{H}
$$

If the $x$-system is of finite character, we have

$$
N\left(A_{x}\right)=\bigcup_{\mathfrak{U} \in \mathfrak{B}} k \mathfrak{U} \mathfrak{U}
$$

Proof. For two bases $\mathfrak{B}_{1}$ and $\mathfrak{B}_{2}$ we get $\bigcup_{\mathfrak{U} \in \mathfrak{B}_{1}} k \mathfrak{U}=\bigcup_{\mathfrak{U} \in \mathfrak{B}_{2}} k \mathfrak{U}$ and the first part follows from Proposition 3. To prove the second half of the lemma, we first observe that $N\left(A_{x}\right)=\varnothing$ implies $\bigcup_{\mathfrak{U} \in \mathfrak{B}} k \mathfrak{H}=\varnothing$. For $a \in \underset{\mathfrak{U} \in \mathfrak{B}}{\bigcup_{x}} k \mathfrak{U}$ we find, by the finite character property, a finite set $\left\{a_{1}, \ldots, a_{n}\right\}$

$\subseteq \bigcup_{\mathfrak{U} \in \mathfrak{B}}^{\mathfrak{U} \in \mathfrak{B}} k \mathfrak{U}$ such that $a \in\left(a_{1}, \ldots, a_{n}\right)_{x}$. Let $a_{i} \in k_{\mathfrak{H}} \mathfrak{H}_{i}$, and determine $\mathfrak{U} \in \mathfrak{B}$ such that $\mathfrak{U} \subseteq \bigcap_{i=1}^{n} \mathfrak{U}_{i}$. Then

$$
a \epsilon \bigcup_{i=1}^{n} k \mathfrak{U}_{i} \subseteq\left(k \bigcap_{i=1}^{n} \mathfrak{U}_{i}\right)_{x}=k \bigcap_{i=1}^{n} \mathfrak{U}_{i} \subseteq k \mathfrak{U} .
$$

We thus have $\bigcup_{\mathfrak{U} \in \mathfrak{B}} k \mathfrak{U} \subseteq \bigcup_{\mathfrak{U} \in \mathfrak{B}} k \mathfrak{U}$, and the lemma is proved.

Obviously $k \mathfrak{I} \subseteq N\left(A_{x}\right) \subseteq A_{x}$. The equalities are reflected in $\mathfrak{I}(S)$ as follows ((1.9) generalizes Theorem 2.7 of [4]).

\section{THEOREM 1.}

(1.8) $\quad N\left(A_{x}\right)=k \mathfrak{I} \Leftrightarrow \overline{\mathfrak{U}}=\mathfrak{I}(S)$ for every neighbourhood $\mathfrak{H}$ of $A_{x}$.

If the $x$-system is of finite character, we get

(1.9) $N\left(A_{x}\right)=A_{x}$ for every $A_{x} \in \mathfrak{I}(S) \Leftrightarrow \mathfrak{F}(a)$ is open for every' $a \in S$.

Proof. Clearly, $N\left(A_{x}\right)=k \mathfrak{I} \Leftrightarrow k \mathfrak{U}=k \mathfrak{T}$ for every neighbourhood $\mathfrak{U}$ of $A_{x}$, and (1.8) follows. Assume that the $x$-system of $S$ is of finite character, and that $N\left(A_{x}\right)=A_{x}$ for every $A_{x} \in \mathfrak{I}(S)$. Then, for $A_{x} \in \mathfrak{F}(a)$, $a \in k \mathfrak{U}_{0}$ for some neighbourhood $\mathfrak{U}_{0}$ of $A_{x}$ (Lemma 1). Thus $A_{x} \in \mathfrak{U}_{0} \subseteq \mathfrak{F}(a)$ and $\mathfrak{F}(a)$ is open. Consverely, assume that $\mathfrak{F}(a)$ is open for every $a \in S^{\prime}$, and let $A_{x} \in \mathfrak{J}(S)$. For $a \in A_{x}$, we get $a \in k \mathfrak{F}(a) \subseteq N\left(A_{x}\right)$ and $A_{x} \subseteq N\left(A_{x}\right)$. Obviously $N\left(A_{x}\right) \subseteq A_{x}$ and (1.9) is proved.

CoRoLlary. Let $\mathbb{S}$ be a commutative semigroup with an $x$-system of finite character, satisfying (0.7). Assume also that $k \mathfrak{X}=O_{x} \neq \varnothing$. Then the structure-space of the minimal prime ideals, $\mathfrak{R}(\mathbb{S})$, is a totally disconnected Hausdorff space.

Proof. Let $P_{x} \in \mathfrak{N}(S)$. Then for every $a \in P_{x}$ there exists by Proposition $0.2, b \in P_{x}$ such that $|a| b \in O_{x}$. For every $Q_{x} \in \mathfrak{N}, b \notin Q_{x} \Rightarrow|a|$ $\epsilon Q_{x} \Rightarrow a \epsilon Q_{x}$, and $a \epsilon k \mathfrak{U}(b) \subseteq N\left(P_{x}\right)$. We conclude $P_{x}=N\left(P_{x}\right)$. As $\mathfrak{N}(\boldsymbol{S})$ is $T_{1}$, the proof is complete.
The connection between the Hausdorff-property of $\mathfrak{I}(S)$ and algebraic properties of $S$ is of the same kind as in the case where $S$ is a ring with the $d$-system. The following theorem is a generalization of Theorem 3.1 of [4].

Theorem 2. (A), (B), (C) and (D) are equivalent.

\section{(A) $\mathfrak{I}(S)$ is a Hausdorff-space.}

(B) For $A_{x}, B_{x}$ different elements of $\Im(S)$, there exists $a \notin A_{x}, b \notin B_{x}$ such that for every $C_{x} \in \mathfrak{I}(S), a \in C_{x} \vee b \in C_{x}$.

(C) For $A_{x}, B_{x}$ different elements of $\mathfrak{I}(S), N\left(A_{x}\right) \nsubseteq B_{x}$.

(D) For every $A_{x} \in \mathfrak{I}(S), N\left(A_{x}\right)$ is contained in exactly one ideal from $\mathfrak{I}$. If $\mathfrak{I} \subseteq \mathfrak{M}$ and the $x$-system is of finite character, (A) is also equivalent to

(E) For $A_{x}, B_{x}$ different ideals from $\mathfrak{I}$, and $a \in S$, there exists $b \in N\left(A_{x}\right)$ such that $a \equiv b\left(B_{x}\right)$.

If every proper ideal in $S$ is contained in an ideal from $\mathfrak{I}$, (A) is also equivalent to

(F) For $A_{x}, B_{x}$ different ideals of $\mathfrak{I}, N\left(A_{x}\right) \cup_{x} N\left(B_{x}\right)=S$.

Proof. Throughout the proof, let $A_{x}, B_{x}$ denote ideals from $\Im$. We first verify $(\mathrm{A}) \Rightarrow(\mathrm{B}) \Rightarrow(\mathrm{C}) \Rightarrow(\mathrm{D}) \Rightarrow(\mathrm{A})$. By Proposition $3,(\mathrm{~A}) \Rightarrow(\mathrm{B})$. Assume (B). Let $A_{x} \neq B_{x}$, and let $a, b$ satisfy (B). Then $b \in k \mathfrak{U}(a) \subseteq N\left(A_{x}\right)$. As $b \in B_{x}$, (C) follows. Obviously (C) $\Rightarrow$ (D). Assume (D). Then, for $A_{x}$ $\neq B_{x}$, Lemma $1,(1.6)$ gives that $\bigcup_{\mathfrak{U} \in \mathfrak{B}} k \mathfrak{U} \nsubseteq B_{x}$ where $\mathfrak{B}$ is the neighbourhood-system of $A_{x}$. Choose $a \in \bigcup_{\mathfrak{U} \in \mathfrak{B}} k \mathfrak{U}, a \notin B_{x}$. Then $a \in k \mathfrak{U}_{0}$ for some $\mathfrak{U}_{0} \in \mathfrak{B}$. Now $B_{x} \in \mathfrak{U}(a)$ and clearly $\mathfrak{H}_{0} \cap \mathfrak{U}(a)=\emptyset$. Thus (A) follows.

Now, assume that the $x$-system is of finite character, and let $\mathfrak{I} \subseteq \mathfrak{M}$. If (A) is satisfied, and $A_{x} \neq B_{x}$, then for $a \in S$ we get two possibilities: for $a \in B_{x}$, choose $b \in N\left(A_{x}\right) \cap B_{x}$. This is possible, for by Theorem 1 , $N\left(A_{x}\right) \neq \varnothing$. Then $\left(a, B_{x}\right)_{x}=\left(b, B_{x}\right)_{x}=B_{x}$ and $a \equiv b\left(B_{x}\right)$. On the other hand, if $a \notin B_{x}$, (C) implies the existence of $b$ such that $b \in N\left(A_{x}\right), b \notin B_{x}$. Here $\left(a, B_{x}\right)_{x}=\left(b, B_{x}\right)_{x}=S$, and $a \equiv b\left(B_{x}\right)$. Assume (E). Let $A_{x} \neq B_{x}$ and choose $a \notin B_{x}$. For $b$ given by $(\mathrm{E}), B_{x} \in \mathfrak{U}(b)$ and $b \in N\left(\boldsymbol{A}_{x}\right)$. By Lemma 1 (1.7), $b \in \mathfrak{k}_{0}$ for some neighbourhood $\mathfrak{U}_{0}$ of $A_{x}$. As $\mathfrak{U}_{0} \cap \mathfrak{U}(b)$ $=\varnothing$, (A) follows.

To prove the last part of the theorem, assume that every proper ideal in $S$ is contained in an ideal from $\mathfrak{J}$. If $-\mathfrak{T}(S)$ is a Hausdorff-space and $A_{x} \neq B_{x}$, (D) implies that $N\left(A_{x}\right) \cup_{x} N\left(B_{x}\right)=S$. If (F) is satisfied, and for some $A_{x} \neq B_{x} N\left(A_{x}\right) \subseteq B_{x}$, we find $B_{x}=S$, in contradiction to the definition of a structure-family. 
LEMMA 2. Assume that the $x$-system is of finite character and that $\mathfrak{I} \subseteq \mathfrak{P}$. Then $N\left(A_{x}\right)$ is half prime for every $A_{x} \in \mathfrak{I}$.

Proof. Let $a \in \operatorname{rad} N\left(A_{x}\right)$, i.e., $a^{n} \in N\left(A_{x}\right)$. For some neighbourhood $\mathfrak{U}_{0}$ of $A_{x}, a^{n} \in k \mathfrak{U}_{0}$ by Lemma 1. For every $B_{x} \in \mathfrak{H}_{0}, a^{n} \in B_{x}$, and since $B_{x} \in \mathfrak{P}$, we have $a \in B_{x}$. Thus $a \in \mathfrak{k H}_{0} \subseteq N\left(A_{x}\right)$.

The following is a generalization of Corollary 3.8 of [4].

THEOREM 3. Assume that the $x$-system is of finite character. Then the following statements are equivalent:

$$
\mathfrak{P}(S) \text { is a Hausdorff-space. }
$$

$\mathfrak{P}(S)$ is totally disconnected.

$$
\text { For every } A_{x} \in \mathfrak{P}, N\left(A_{x}\right)=A_{x} \text {. }
$$

Proof. We verify $(C) \Rightarrow(B) \Rightarrow(A) \Rightarrow(C)$. (C) $\Rightarrow(B)$ follows from Theorem $1(B),(B) \Rightarrow(A)$ is obvious. Assume (A). By the Krull-Stone theorem, Lemma 2 gives

$$
N\left(A_{x}\right)=\overbrace{\left\{P_{x} \in \mathfrak{P} \mid P_{x} \supseteq N\left(A_{x}\right)\right\}} P_{x}
$$

If $N\left(A_{x}\right) \neq A_{x}$, then for some $P_{x} \neq A_{x}, P_{x} \supseteq N\left(A_{x}\right)$, contradicting (A).

§ 2. Compactness. We now turn to the compactness of structurespaces, and make the following observation:

Proposition 4. $\{\mathfrak{U}(a)\}_{a \in R}$ is an open covering of $\mathfrak{I}(S)$ if and only if $R \nsubseteq A_{x}$ for every $A_{x} \in \mathfrak{I}$.

THEOREM 4. $\mathfrak{I}(S)$ is compact if and only if every subset $R$ of $S$ with the property $R \nsubseteq A_{x}$ for every $A_{x} \in \mathfrak{I}$, contains a finite subset $N$ with the property $N \nsubseteq A_{x}$ for every $A_{x} \in \mathfrak{I}$.

Proof. The theorem follows from Proposition 3 and Proposition 4.

THEOREM 5. If the $x$-system is of finite character, $\mathfrak{I}(S)$ is compact if and only if every ideal $R_{x}$ which satisfies $R_{x} \nsubseteq A_{x}$ for every $A_{x} \in \mathfrak{I}$, contains a finitely generated ideal $N_{x}$ such that $N_{x} \nsubseteq A_{x}$ for every $A_{x} \in \mathfrak{I}$.

Proof. The necessity of the condition follows from Theorem 4. Assume the condition. Let $R \subset S$ satisfy $R \nsubseteq A_{x}$ for every $A_{x} \in \mathfrak{T}$. Then also $R_{x} \nsubseteq A_{x}$ for every $A_{x} \in \bar{I}$ and by the condition we find a finitely generated ideal $N_{x} \subseteq R_{x}$ such that $N_{x} \nsubseteq A_{x}$ for every $A_{x} \in \mathfrak{I}$. By the finite character property we find for every $a \in N$ an $F_{a} \subseteq R$ such that $a \in\left(F_{a}\right)_{x}, F_{a}$ finite for every $a \in N$. As $N$ is finite, $F=\bigcup_{a \in N} F_{a}$ is finite, and $N_{x} \subseteq F_{x}$. Clearly $F_{x} \nsubseteq A_{x}$ for every $A_{x} \in \mathfrak{I}$, thus also $F \nsubseteq A_{x}$ for every $A_{x} \in \mathfrak{J}$, and as $F \subseteq R, \mathfrak{I}(S)$ is compact by Theorem 4 .

COROLLARY. If the $x$-system is of finite character, and if every proper ideal in $\mathbb{S}$ is contained in an ideal from $\mathfrak{J}$, then $\mathfrak{I}(\mathcal{S})$ is compact if and only if $S$ is finitely generated.
Proof. Under the assumptions of the corollary, $N_{x} \nsubseteq A_{x}$ for every $A_{x} \in \mathfrak{I}$, if and only if $N_{x}=S$.

The compactness of $\mathfrak{I}(S)$ thus amounts to a finiteness condition on $S$. On the other hand, we may take a somewhat different point of view:

A proper subset $V$ of $S$ is said to be an $f$-set for $\mathfrak{I}$ if for every finite subset $N$ of $V$ there exists an $A_{x} \in \mathfrak{I}$ such that $N \subseteq A_{x}$ (see [ $\left.\tilde{\sigma}\right]$ ). By Zorn's Lemma we find that every $f$-set for $\mathfrak{I}$ in $S$ is contained in a maximal $f$-set for $\mathfrak{I}$ in $S$.

THEOREM 6. $\mathfrak{I}(S)$ is compact if and only if every maximal $f$-set for $\mathfrak{I}$ is a member of $\mathfrak{\Im}$.

Proof. Assume that $\mathfrak{I}(S)$ is compact, and let $W$ be a maximal $f$-set. We contend that $W \in \mathfrak{I}$. As every $A_{x} \in \mathfrak{I}$ is an $f$-set, it is sufficient to find $A_{x} \in \mathfrak{I}$ with $A_{x} \supseteq W$. Assume that $A_{x} \nsubseteq W$ for every $A_{x} \epsilon \mathfrak{I}$. By Theorem 4 we find a finite subset $N$ of $W$ with $N \nsubseteq A_{x}$ for every $A_{x} \in \mathfrak{I}$, a contradiction. Conversely, assume that every maximal $f$-set is an element of $\mathfrak{I}$. If $\mathfrak{I}(S)$ were not compact, we could find an $f$-set $R$ for $\mathfrak{I}$ with $R \nsubseteq \nsubseteq A_{x}$, in contradiction to the assumption.

§ 3. Disconnected $\mathfrak{M}(S)$. Let $R$ be a commutative ring with identity. If $R$ is semisimple, then $\mathfrak{M}(R)$ is disconnected if and only if $R$ is the direct sum of two of its proper ideals $R_{1}$ and $R_{2}$. If $\mathfrak{M}(R)$ $=\mathfrak{F}_{1} \cup \mathfrak{F}_{2}$ is a partition of $\mathfrak{M}(R)$ into disjoint open-closed proper subsets, we may determine $R_{1}$ and $R_{2}$ so that $\mathfrak{M}\left(R_{i}\right)$ is homeomorphic to $\mathfrak{F}_{i}$, $i=1,2$. (See for instance [6].)

We observe that if $R$ is the direct sum of the ideals $R_{1}$ and $R_{2}$, then $R=R_{1} \cup_{d} R_{2}, R_{1} \cap R_{2}=\{0\}$ and every $d$-ideal $A$ of $R_{i}$ is a $d$-ideal of $R$. In view of this the following theorem generalizes the above-mentioned theorem for rings.

THEOREM 7. Let $S$ be a commutative semigroup with an $x$-system of finite character and $x$-identity. Then $\mathfrak{M}(S)$ is disconnected if and only if there exist ideals $A_{x}, B_{x}$ in $S$, different from $S$ and $k \mathfrak{M}$, such that $A_{x} \cup_{x} B_{x}$ $=S, A_{x} \cap B_{x}=k \mathfrak{M}$.

If the $x$-system is additive, and if $k \mathfrak{M}=k \mathfrak{X}=O_{x}$, then for every partition of $\mathfrak{M}(S)$ into disjoint open-closed proper subsets $\mathfrak{A}$ and $\mathfrak{B}$, we may determine $A_{x}$ and $B_{x}$ such that $\mathfrak{M}\left(A_{x}\right)$ is homeomorphic to $\mathfrak{A}$ and $\mathfrak{M}\left(B_{x}\right)$ homeomorphic to $\mathfrak{B}$, where $A_{x}$ and $B_{x}$ are equipped with the $x$-systems induced from $\mathrm{S}$.

Proof. Let $\mathfrak{M}(S)=\mathfrak{A} \cup \mathfrak{B}$. be a partition of $\mathfrak{M}(S)$ into proper, disjoint open-closed subsets and put $A_{x}=k \mathfrak{B}, B_{x}=k \mathfrak{A}$. Then $A_{x} \cap B_{x}$ $=k \mathfrak{M}$. Furthermore, $A_{x} \cup_{x} B_{x}=S$, for if $A_{x} \cup_{x} B_{x}$ were a proper ideal, it would be contained in some maximal ideal $M_{x}$. (This is proved in the usual way by the existence of an $x$-identity.) Then $M_{x} \in \mathfrak{A} \cap \mathfrak{B}$, a contradiction. Clearly $A_{x}$ and $B_{x}$ are different from $S$ and $k \mathfrak{M}$. 
Conversely, assume the existence of $A_{x}$ and $B_{x}$ satisfying the condition of the theorem. Put $\mathfrak{A}=h B_{x}, \mathfrak{B}=h A_{x}$. Clearly, $\mathfrak{A}$ and $\mathfrak{B}$ are closed. Since $\mathfrak{M} \subset \mathfrak{B}$ and $A_{x} \cap B_{x}=k \mathfrak{M}$, we have $\mathfrak{A} \cup \mathfrak{B}=\mathfrak{M}(S)$. Furthermore $A_{x} \cup_{x} B_{x}=S$ implies $\mathfrak{A} \cap \mathfrak{B}=0$, and the first part of the theorem is proved.

Finally assume the condition of the last part of the theorem. Define $A_{x}$ and $B_{x}$ as above, $A_{x}=k \mathfrak{B}, B_{x}=k \mathfrak{A}$, where $\mathfrak{U} \cup \mathfrak{B}$ is a partition of $\mathfrak{M}(S)$ into proper, disjoint open-closed subsets. By symmetry it is sufficient to prove that $\mathfrak{M}\left(A_{x}\right)$ is homeomorphic to $\mathfrak{A}$. We denotie the $x$-system induced on $A_{x}$ from $S$ by $x_{1}$, and prove first that $A_{x} / O_{x}$ is isomorphic to $S / B_{x}$. Denote the canonical mapping of $A_{x}$ ontio $A_{x} / O_{x}$ by $\varphi$, of $S$ onto $S / B_{x}$ by $\psi$; and denote the canonical $x$-system of $S / B_{x}$ by $\bar{x}$, of $A_{x} / O_{x}$ by $\bar{x}_{1}$. Now define

$$
\bar{\psi}: A_{x} / O_{x} \rightarrow S / B_{x}
$$

by $\bar{\psi}(\varphi(s))=\psi(s)$ for $s \in A_{x}$. By definition $A_{x} / O_{x}=A_{x} / A_{x} \cap B_{x}, S / B_{x}$ $=A_{x} \cup_{x} B_{x} / B_{x}$, and $\bar{\psi}$ is bijective by Theorem 0.2 . Clearly $\bar{\psi}$ is multiplicative, and every $\bar{x}_{1}$-ideal in $A_{x} / O_{x}$ may be written in the form $p\left(C_{x_{1}}\right)$, where $C_{x_{1}}$ is some $x_{1}$-ideal in $A_{x}$, i.e., an $x$-ideal contained in $A_{x}$. Now $\bar{\psi}\left(\varphi\left(C_{x_{1}}\right)\right)=\psi\left(C_{x_{1}} \cup_{x} A_{x}\right)$, showing that the image by $\bar{\psi}$ of every $\bar{x}_{1}$-ideal in $A_{x} / O_{x}$ is an $\bar{x}$-ideal in $S / B_{x}$. Conversely, every $\bar{x}$-ideal in $S / B_{x}$ may be written as $\psi\left(D_{x}\right)$, where $D_{x} \supseteq B_{x}$. Now

$$
\begin{aligned}
\bar{\psi}^{-1}\left(\psi\left(D_{x}\right)\right) & =\left\{\varphi(s) ; s \in A_{x} \text { and } \psi(s) \in \psi\left(D_{x}\right)\right\} \\
& =\left\{\varphi(s) ; s \in A_{x} \text { and } s \in D_{x}\right\}=\varphi\left(A_{x} \cap D_{x}\right)
\end{aligned}
$$

showing that $\bar{\psi}$ is an isomorphism. This shows in particular that $A_{x} / O_{x}$ has an $x$-identity, so that the maximal ideals is a structure-family of $A_{x} / O_{x}$. Furthermore,

$$
\mathfrak{M}\left(A_{x} / O_{x}\right) \text { is homeomorphic to } \mathfrak{M}\left(S / B_{x}\right) \text {. }
$$

By (1.2) and (1.3) the family of maximal ideals in $A_{x}$ is a structurefamily, and

$$
\text { (3.2) } \mathfrak{M}\left(A_{x}\right) \text { is homeomorphic to } \mathfrak{M}\left(A_{x} / O_{x}\right) \text {. }
$$

Finally, the corollary of Proposition 2 gives

$$
\mathfrak{M}\left(S / B_{x}\right) \text { is homeomorphic to } \mathfrak{A} \text {. }
$$

(3.1), (3.2) and (3.3) implies that $\mathfrak{M}\left(A_{x}\right)$ is homeomorphic to $\mathfrak{A}$, and the theorem is proved.

§ 4. A representation theorem. The $l$-system of a distributive lattice $L$ is a half prime $x$-system of finite character, when $L$ is considered as a semi-group under $\wedge$. This $x$-system has the property that every finitely generated ideal is a principal ideal. We shall now prove a theorem closely related to a converse of this statement. In fact, let $S$ be a commutative semigroup with a half-prime $x$-system of finite character, where every finitely generated ideal is a principal ideal. Denote the canonical $x$-system of $S / O_{x}$ by $\bar{x}$. Then we have:

THEOREM 8. There exists a family $\mathfrak{L}$ of open sets in $\mathfrak{P}(S)$ such that $\mathfrak{L}$ is a lattice under $\cap$ and $\cup$, and such that $\mathbb{S} / O_{x}$ is $(\bar{x}, l)$-isomorphic to $\mathfrak{Q}$, where $\mathfrak{L}$ is considered as a semigroup under $\cap$.

Proof. Put $\mathfrak{L}=\{\mathfrak{U}(s) ; s \in S\}$. For $s_{1}, s_{2} \in S$ there exists $s \in S^{\prime}$ such that $(s)_{x}=\left(s_{1}, s_{2}\right)_{x}$. Then

since $\mathfrak{P}(S)$ consists of prime ideals,

$$
\mathfrak{U}\left(s_{1}\right) \cap \mathfrak{U}\left(s_{2}\right)=\mathfrak{U}\left(s_{1} s_{2}\right)
$$

and $\mathfrak{L}$ is a lattice under $\cap$ and $\cup$. For $\bar{s} \in S / O_{x}$, put $\varphi(\bar{s})=\mathfrak{U}(s)$. Now, $\bar{s}_{1}=\bar{s}_{2}$ if and only if $\left(s_{1}, O_{x}\right)_{x}=\left(s_{2}, O_{x}\right)_{x}$. The $x$-system is half prime, so this is equivalent to $k \mathfrak{F}\left(s_{1}\right)=k \mathfrak{F}\left(s_{2}\right)$, which is again equivalent to $\mathfrak{F}\left(s_{1}\right)=\mathfrak{F}\left(s_{2}\right)$. To sum up, $\bar{s}_{1}=\bar{s}_{2} \Leftrightarrow \mathfrak{U}\left(s_{1}\right)=\mathfrak{U}\left(s_{2}\right)$. This shows that $\varphi$ is well defined and injective. Clearly $\varphi$ is surjective; that $\varphi$ is multiplicative follows by (4.2), and it remains to be shown that $\varphi$ establishes a 1-1 correspondence between the ideals in $S / O_{x}$ and $\mathbb{L}$. To every $\bar{x}$-ideal $A_{\bar{x}}$ in $S / O_{x}$ there corresponds an $x$-ideal $B_{x}$ in $S$ such that $A_{\bar{x}}=\psi\left(B_{x}\right)$, where $\psi$ is the canonical mapping of $S$ onto $S / O_{x}$. Now $\varphi\left(A_{-x}\right)=\{\mathfrak{U}(s)$; $\left.s \in B_{x}\right\}$. For $s \in S, s_{1} \in B_{x}$, we find $\mathfrak{U}(s) \cap \mathfrak{U}\left(s_{1}\right)=\mathfrak{U}\left(s_{1} s\right) \in \varphi\left(A_{\bar{x}}\right)$. For $s_{1}, s_{2} \in B_{x}$ we find $s \in \mathbb{S}$ such that $(s)_{x}=\left(s_{1}, s_{2}\right)_{x}$, and $\mathfrak{U}\left(s_{1}\right) \cup \mathfrak{U}\left(s_{2}\right)=\mathfrak{U}(s)$ $\epsilon \varphi\left(A_{\bar{x}}\right)$, since we clearly have $s \in B_{x}$. Thus $\varphi\left(A_{\bar{x}}\right)$ is an $l$-ideal. Conversely, every $l$-ideal in $\mathfrak{I}$ may be written in the form $\{\mathfrak{U}(a) ; a \in A\}$ for some $A \subseteq S$. As $\varphi^{-1}\left(\left\{\mathfrak{U}(a) ; a \in A_{x}\right\}\right)=\psi\left(A_{x}\right)$, the proof is complete if we show that $A_{x}=A$. It is sufficient to show that for any finite subset $N$ of $A, N_{x} \subseteq A$. To see this, we observe that if $N_{x}=(s)_{x}$, then $\bigcup_{a \in N} \mathfrak{U}(a)=\mathfrak{U}(s)$, and as $\{\mathfrak{U}(a) ; a \in A\}$ is an $l$-ideal, $\mathfrak{U}(s) \in\{\mathfrak{U}(a) ; a \in A\}$, $s \in A$ and for every $t \in(s)_{x}$, we have $s \in P_{x} \Rightarrow t \in P_{x}$ for every prime ideal $P_{x}$. Thus $\mathfrak{U}(t) \subseteq \mathfrak{U}(s)$, and again since $\{\mathfrak{U}(a) ; a \epsilon A\}$ is an $l$-ideal, we conclude that $t \in A$. To sum up, $N_{x} \subseteq A$, and the proof is complete.

§5. A characteristic property for Boolean algebras. Finally we give an application of the previous theory to a simple algebraic problem. Let $L$ be a distributive lattice with the $l$-system.

THEOREM 9. $\mathfrak{P}(L)$ is compact if and only if $L$ has a greatest element.

Proof. Since the $l$-system is half-prime of finite character, and every finitely generated ideal is a principal ideal, the theorem follows from the corollary of Theorem 5 . 
THEOREM 10. Let $I$ be a distributive lattice with a greatest element $I$ and a least element 0 . Then $L$ is a Boolean algebra if and only if $\mathfrak{P}(L)$ is Hausdorff.

Proof. If $L$ is a Boolean algebra, then for any $a \in L, \mathfrak{U}(a)=\mathfrak{F}(\mathrm{C} a)$, and $\mathfrak{P}(L)$ is Hausdorff by Theorem 1 and Theorem $3(\mathrm{C})$. Conversely, assume that $\mathfrak{P}(L)$ is Hausdorff and let $a \neq 0, I$. Let $P_{l} \notin \mathfrak{F}(a)$. For every $Q_{l} \in \mathfrak{F}(a)$ there exists an element $d$ such that $Q_{l} \in \mathfrak{U}(d)$ and $P_{l} \notin \mathfrak{U}(d)$. These $\mathfrak{U}(d)$ form an open covering of the compact set $\mathfrak{F}(a)$ (Theorem 9 ), and we can find $d_{1}, d_{2}, \ldots, d_{n}$ such that

$$
\mathfrak{F}(a) \subseteq \mathfrak{U}\left(d_{1}\right) \cup \ldots \cup \mathfrak{U}\left(d_{n}\right)=\mathfrak{U}\left(\bigvee_{i=1}^{n} d_{i}\right)
$$

Now put $b=\bigvee_{i=1}^{n} d_{i}$. By Theorem $3(\mathrm{C})$ and Theorem $1, \mathfrak{F}(b)$ is open, and $P_{l} \in \mathfrak{F}(b)$. On the other hand $\mathfrak{U}(a)$ is closed and therefore compact. Thus the sets $\mathfrak{F}(b)$ form an open covering of the compact set $\mathfrak{I}(a)$, and we can find $b_{1}, \ldots, b_{m}$ such that

$$
\mathfrak{H}(a) \subseteq \mathfrak{F}\left(b_{1}\right) \cup \mathfrak{F}\left(b_{2}\right) \cup \ldots \cup \mathfrak{F}\left(b_{m}\right)=\left(\bigwedge_{i=1}^{m} b_{i}\right)
$$

Now, for every $P_{l} \in \mathfrak{P}, a \wedge\left(\bigwedge_{i=1}^{m} b_{i}\right) \in P_{l}$, and since $k_{\mathfrak{P}}=\{0\}$ we have $a \wedge\left(\bigwedge_{i=1}^{m} b_{i}\right)=0$. On the other hand, $a \vee\left(\bigwedge_{i=1}^{m} b_{i}\right)=\bigwedge_{i=1}^{m}\left(a \vee b_{i}\right)$ is contained in no proper prime ideal. In fact, if $\bigwedge_{i=1}^{m}\left(a \vee b_{i}\right) \in P_{l}$, then for some $i_{0}$, $a \vee b_{i_{0}} \in P_{l}$, consequently $a \in P_{l}$ and $b_{i_{0}} \in P_{l}$, i.e., $P_{l} \in \mathfrak{F}(a)$ and $P_{l} \notin \mathfrak{U}\left(b_{i_{0}}\right)$, which is impossible. This gives that $a \vee\left(\bigwedge_{i=1}^{m} b_{i}\right)=I$, and $\bigwedge_{i=1}^{m} b_{i}$ is a complement of $a$.

CoRoLlary (Nachbin). A distributive lattice with 0 and $I$ is a Boolean algebra if and only if every proper prime ideal is a maximal ideal.

Proof. For a distributive lattice we always have $\mathfrak{M} \subset \mathfrak{P}$. If $\mathfrak{P}(L)$ is Hausdorff, we therefore conclude $\mathfrak{M}=\mathfrak{P}$. On the other hand, if $\mathfrak{M}=\mathfrak{P}$, then clearly $\mathfrak{M}=\mathfrak{N}=\mathfrak{P}$, and by the corollary of Theorem $1, \mathfrak{P}(L)$ is Hausdorff.

\section{References}

[1] K. E. Aubert, Theory of $x$-ideals, Acta Math. 107 (1962), pp. 1-52.

[2] - Additive ideal systems and commutative algebra, Mat. Seminar no. 3, Univ. of Oslo, 1963 .

[3] R. L. Blair and C. L. Eggan, On the compactness of the strueture-space of a ring, Proc. Amer. Math. Soc., 11 (1960), pp. 876-879. pp. 1-16.

[4] L. Gillman, Rings with Hausdorff structure space, Fund. Math. 45 (1958),

[5] J. G. Horne, On the ideal structure of certain semi-rings and compactification of topological spaces, Trans. Amer. Math. Soc. 90 (1959), pp. 408-430.

[6] N. Jacobson, $A$ topology for the set of primitive ideals in an arbitrary ring, Proc. Nat. Acad. of Sciences 31 (1945), pp. 333-338.

[7] D. G. Johnson and J. E. Kist, Prime ideals in vector lattices, Can. Journal of Math. 14 (1962), pp. 349-354.

[8] C. W. Kohls, The space of prime ideals of a ring, Fund. Math. 45 (1958), pp. 17-27.

[9] L. N a ch bin, Une propriété charactéristique des algèbres booleiennes, Portugalie Math. 6 (1947), pp. 115-118.

[10] W. Słowikowski and W. Zawadowski, A generalization of maximal ideal method of Stone and Gelfand, Fund. Math. 42 (1955), pp. 215-231.

UNIVERSITY OF OSLO, NORWAY

Regu par la Rédaction le 31.12.1964 